\title{
Physical Activity, Sedentary Behavior, and Time in Bed Among Finnish Adults Measured 24/7 by Triaxial Accelerometry
}

\author{
Pauliina Husu, Kari Tokola, \\ Henri Vähä-Ypyä, \\ Harri Sievänen, and Jaana Suni \\ UKK Institute for Health Promotion \\ Research
}

\author{
Kaisu M. Kaikkonen \\ Oulu Deaconess Institute \\ Foundation sr
}

\author{
Olli J. Heinonen
University of Turku \\ Olli J. Heinonen
University of Turku
}

Kai Savonen

Kuopio Research Institute of Exercise

Medicine and Kuopio University

Hospital

\author{
Jarmo Heiskanen \\ LIKES Research Centre for Physical \\ Activity and Health
}

\section{Tommi Vasankari}

UKK Institute for Health Promotion Research and Tampere University

\begin{abstract}
Background: Studies measuring physical activity (PA) and sedentary behavior on a $24 / 7$ basis are scarce. The present study assessed the feasibility of using an accelerometer at the hip while awake and at the wrist while sleeping to describe 24/7 patterns of physical behavior in working-aged adults by age, sex, and fitness. Methods: The study was based on the FinFit 2017 study where the physical behavior of 20- to 69-year-old Finns was assessed 24/7 by triaxial accelerometer (UKKRM42; UKK Terveyspalvelut Oy, Tampere, Finland). During waking hours, the accelerometer was kept at the right hip and, during time in bed, at the nondominant wrist. PA variables were based on 1-min exponential moving average of mean amplitude deviation of the resultant acceleration signal analyzed in 6-s epochs. The angle for the posture estimation algorithm was used to identify sedentary behavior and standing. Evaluation of time in bed was based on the wrist movement. Fitness was estimated by the 6-min walk test. Results: A total of 2,256 eligible participants (mean age 49.5 years, $S D=13.5,59 \%$ women) wore the accelerometer at the hip $15.7 \mathrm{hr} /$ day $(S D=1.4)$ and at the wrist $8.3 \mathrm{hr} /$ day $(S D=1.4)$. Sedentary behavior covered $9 \mathrm{hr} 18 \mathrm{~min} /$ day $(S D=1.8 \mathrm{hr} / \mathrm{day})$, standing nearly $2 \mathrm{hr} /$ day $(S D=0.9)$, light PA $3.7 \mathrm{hr} /$ day $(S D=1.3)$, and moderate to vigorous PA $46 \mathrm{~min} /$ day $(S D=26)$. Participants took 7,451 steps per day $(S D=2,962)$ on average. Men were most active around noon, while women had activity peaks at noon and at early evening. The low-fit tertile took 1,186 and 1,747 fewer steps per day than the mid- and high-fit tertiles (both $p<.001$ ). Conclusions: One triaxial accelerometer with a two wear-site approach provides a feasible method to characterize hour-by-hour patterns of physical behavior among working-aged adults.
\end{abstract}

Keywords: device-based, movement, sleep, standing

Some years ago, discussion about the importance of including 24-hr movement behavior in health research was initiated (e.g., Chaput, Carson, Gray, \& Tremblay, 2014). The time-use paradigm was presented as a multidisciplinary approach to

Husu, Tokola, Vähä-Ypyä, Sievänen, Suni, and Vasankari are with the UKK Institute for Health Promotion Research, Tampere, Finland. Heinonen is with the Paavo Nurmi Centre \& Unit for Health and Physical Activity, University of Turku, Turku, Finland. Heiskanen is with the LIKES Research Centre for Physical Activity and Health, Jyväskylä, Finland. Kaikkonen is with the Department of Sports and Exercise Medicine, Oulu Deaconess Institute Foundation sr, Oulu, Finland. Savonen is with the Kuopio Research Institute of Exercise Medicine, Kuopio, Finland; and the Department of Clinical Physiology and Nuclear Medicine, Kuopio University Hospital, Kuopio, Finland. Kokko is with the Faculty of Sport and Health Sciences, University of Jyväskylä, Jyväskylä, Finland. Vasankari is also with the Faculty of Medicine and Health Technology, Tampere University, Tampere, Finland Husu (pauliina.husu@ukkinstituutti.fi) is corresponding author. investigate the allocation of time within the given time period (Pedisic, Dumuid, \& Olds, 2017). As moderate to vigorous physical activity (MVPA), light physical activity (PA), sedentary behavior (SB), and sleep or time in bed (TIB) consume basically the entire 24hr time of the day, they are the behaviors that are recommended to be assessed within the context of the time-use paradigm as additive and interactive risk factors for health and disease (Pedisic et al., 2017; Rosenberger et al., 2019; Sievänen \& Kujala 2017).

The PA is well-known to be closely related to cardiorespiratory fitness (CRF; Kujala et al., 2017), while high CRF is associated with better cardio-metabolic health and lower mortality even more strongly than PA (Lee et al., 2011). However, the number of population-based studies including both the assessment of CRF and device-based PA are yet scarce (Schram et al., 2014; Vasankari et al., 2017; Zou et al., 2019).

So far, only a few population-based studies have utilized the 24-hr approach to analyze PA, SB, and sleep/TIB (Cabanas- 
Sánchez et al., 2020; Doherty et al., 2017; Koolhaas et al., 2017; Niemelä et al., 2019; Ramires et al., 2017; Schram et al., 2014; Wennman et al., 2019). As measurement methods, these studies have employed either wrist-worn (Cabanas-Sánchez et al., 2020; Doherty et al., 2017; Koolhaas, et al., 2017; Niemelä et al., 2019; Ramires et al., 2017; Wennman et al., 2019) or thigh-worn accelerometers (Schram et al., 2014). For practical reasons, it is not possible, in large population-based studies, to use several accelerometers attached to the body. Yet there is no consensus of which single accelerometer setup would adequately capture the entire 24-hr spectrum of PA, SB, and TIB.

Wrist-worn devices are ideal for large-scale data collection and sleep analysis, but they are not able to identify body posture (Rosenberger et al., 2019), a fact that fundamentally compromises the SB measurement. Thigh-worn devices, in turn, are also widely used and reliable in recognizing both body posture and intensity of PA (Crowley et al., 2019; Lyden, Kozey-Keadle, Staudenmayer, \& Freedson, 2017; van der Velde et al., 2017), but their utility in sleep assessment remains questionable. The hip-worn accelerometer is not ideal for sleep assessment either, but it can capture both locomotion and most other large body movements in various activities with better accuracy than the wrist-worn devices (Rosenberger et al., 2013). However, hip-worn accelerometers have generally measured PA and $\mathrm{SB}$ as counts per minute and employed count-based thresholds for separating PA from SB, which makes these methods unable to distinguish between stationary postures, like lying, sitting, and standing, nor identify patterns in them accurately (Barreira, Zderic, Schuna, Hamilton, \& TudorLocke, 2015; Judice, Santos, Hamilton, Sardinha, \& Silva, 2015). Employing raw acceleration data and the coincident orientation of the accelerometer, in terms of Earth's gravity vector instead of count values and concomitant intensity thresholds, a triaxial accelerometer worn at the hip can identify temporal patterns in lying, sitting, standing, sit-to-stand transitions, and PA performed at different intensity categories with high accuracy (Vähä-Ypyä et al., 2015; Vähä-Ypyä, Husu, Suni, Vasankari, \& Sievänen, 2018).

Thus, the hip is considered the site of choice for PA and SB measurement during waking hours, whereas the wrist is recommended for TIB measurement (Sievänen \& Kujala, 2017). With adjustable hip and wrist bands, a single triaxial accelerometer can be used for all these measurements.

Previous studies (e.g., Cabanas-Sánchez et al., 2020; Husu et al., 2016; Niemelä et al., 2019; Ramires et al., 2017; Wennman et al., 2019) have used different accelerometer brands and different analysis algorithms to determine the duration and intensity of PA, which makes the comparison of PA results from different studies difficult (Chaudhry et al., 2020). Steps represent a fundamental metric of human locomotion (Bassett, Toth, LaMunion, \& Crouter, 2017), and they are strongly associated with health and mortality (e.g., Bassett et al., 2017; Saint-Maurice et al., 2020). Step number is also a practical and easily understandable indicator of PA and is thus proposed as a preferred metric for quantifying PA in general (Bassett et al., 2017). A recent meta-analysis of PA interventions also pinpoints the importance of step monitoring as a means to address the public health challenge pertaining to physical inactivity (Chaudhry et al., 2020).

The present population-based study employed the same triaxial accelerometer worn at the hip during waking hours and at the wrist while in bed for sleep, the measurement covering the entire 24-hr time period per day. As both age and sex are known to be associated with PA and SB (e.g., Cabanas-Sánchez et al., 2020; Doherty et al., 2017; Husu et al., 2016; Niemelä et al., 2019; Ramires et al., 2017; Wennman et al., 2019), the purpose of the present study was to assess the feasibility of using this two wear-site approach to describe (a) sex- and age-group differences in daily PA, SB, standing, and TIB and (b) hour-byhour patterns of SB, steps, and MVPA among Finnish workingaged adults during the $24 / 7$ measurement period. Furthermore, participants' fitness levels were taken into account when illustrating the PA patterns.

\section{Methods}

This study is based on the population-based FinFit 2017 study, which is a multifactorial study on PA, fitness, and health conducted with a stratified random sample of 20- to 69-year-old Finnish adults $(n=13,500)$. The primary purpose of the FinFit 2017 study was to measure 24/7 movement spectrum and physical fitness of the target group. Potential participants were drawn from the population registry in seven city-centered regions of Finland: 300 men and women from both Helsinki and Tampere regions and 150 men and women from each of Turku, Kuopio, Jyväskylä, Oulu, and Rovaniemi regions spread across five age groups (20-29, 30-39, 40-49, 50-59, and 60-69 years). Other inclusion or exclusion criteria were not used. Invitation letters containing information about the study and informed consent with the option to withdraw from the study at any time were mailed to all 13,500 potential participants belonging to the sample. The data collection was conducted between September 2017 and March 2019.

The study was composed of three parts: (a) a questionnaire assessing health status and health-related behaviors, including PA; (b) health examination, including blood samples and anthropometric measurements and health-related fitness tests (shoulder-neck mobility, modified push-up [Suni et al., 1996] and a 6-min walk test [Mänttäri et al., 2018]); and (c) 24/7 accelerometer measurement with a triaxial accelerometer.

Participation in the study was voluntary. The Regional Ethics Committee of the Expert Responsibility area of Tampere University Hospital approved the study (R17030). All participants gave signed informed consent before participation.

\section{Accelerometer Measurements}

During waking hours, the accelerometer (UKK RM42; UKK Terveyspalvelut $\mathrm{Oy}$ ) was attached to an elastic belt and worn on the right side of the hip for seven consecutive days, except during shower and other water activities. When going to bed for sleep, the participant moved the accelerometer from the belt to an adjustable wrist band and attached it to the nondominant wrist on the knuckles side. Participants received both oral and written instructions about accelerometer use and how to change the attachment point.

The accelerometer collects and stores the raw triaxial data in actual $g$ units in $\pm 16 g$ range at the $100 \mathrm{~Hz}$ sampling rate. The accelerometer is initialized so that it starts collecting data if the absolute value of the difference between a reference value and the incident acceleration exceeds $187.5 \mathrm{mg}$ in any axis and, within the next $5 \mathrm{~s}$, the difference exceeds $500 \mathrm{mg}$ in any axis; if not, the accelerometer returns to quiescent state (Caramaliu, Vasile, \& Bacis, 2015). Whenever the raw acceleration in any axis exceeds the previous limits, the reference values are updated with the incident acceleration values. If continuous quiescent time is longer than 120 min (Jaeschke et al., 2017), this period is considered nonwear time. Otherwise, the quiescent time denotes stationary 
time. In case of daily nonwear time, the given day was excluded from the data. After the measurement, the collected acceleration data were saved to hard disk for further analysis. The analysis was performed with the Microsoft Excel Visual Basic for Applications program (2016; Microsoft Corporation, Santa Rosa, CA).

The mean amplitude deviation (MAD) was determined from the resultant acceleration of three orthogonal acceleration components in 6-s epochs, which has been found to be a valid $\left(R^{2}=.94\right)$ indicator of incident oxygen consumption during locomotion (Vähä-Ypyä et al., 2015). The epoch-wise MAD values were converted to metabolic equivalent of task (METs; $3.5 \mathrm{ml} \cdot \mathrm{kg}^{-1} \cdot \mathrm{min}^{-1}$ of oxygen consumption), and intensity was calculated as the 1-min exponential moving average of epochwise MET values. Light PA was defined as MET value higher than or equal to 1.5 and $<3.0$ (MAD value between 22.5 and $91.5 \mathrm{mg}$ ) and MVPA as MET value higher than or equal to 3.0 (MAD over $91.5 \mathrm{mg}$ ) (Vähä-Ypyä et al., 2015). The body posture was determined only for epochs, when the MAD value was lower than $22.5 \mathrm{mg}$ (Vähä-Ypyä et al., 2018).

In line with the definition of SB (Tremblay et al., 2017), time spent in sitting and reclining positions without movement $(<1.5$ METs) were combined to indicate SB, whereas standing was analyzed separately. The 6-s epoch-wise values representing SB or standing periods were also smoothed by a 1-min exponential moving average. The determination of the body posture was based on two facts: the Earth's gravity vector is constant, and the body posture during walking is upright. The accelerometer orientation, in terms of the gravity vector during walking, was taken as the reference, and the angle for posture estimation was determined from the concurrent accelerometer orientation in relation to the reference vector (Vähä-Ypyä et al., 2018). In standardized conditions, standing could be separated from sitting or lying with $100 \%$ accuracy and sitting from lying with $95 \%$ accuracy. In free-living conditions, the agreement between the posture classification from simultaneous thigh- and hip-worn data was about 90\% (Vähä-Ypyä et al., 2018).

In the present study, TIB was used as a crude indicator of sleep-related behavior, and the analysis was based on the movement of the nondominant wrist during the hours when the accelerometer was kept on the wrist band. The bed-in time (time going to bed for sleeping) was defined as the moment when the accelerometer was moved from the hip to the wrist and the bed-out time (time to wake up) when it was moved from the wrist to the hip. Identification of the bed-in and bed-out times was based on the idea that it is easier to turn the accelerometer upside down on the wrist than on the hip. The sensor location was determined from the amount and frequency of changes in the accelerometer orientation. The method used for differentiating the sensor location classifies the sensor location between the hip and wrist. The 6-s epoch-wise axis-specific mean values of the acceleration data are stored in a vector, and the angle between the adjacent vectors is calculated. The number of over $5^{\circ}, 15^{\circ}$, and $60^{\circ}$ angles and their ratios within a window looking $35 \mathrm{~min}$ forward and $35 \mathrm{~min}$ backward are used as input values for a binary logistic regression model. The model output values less than 0.5 denote the wrist location and higher than 0.5 the hip location. The TIB classification was based on the number of at least $5^{\circ}$ changes in accelerometer orientation between two consecutive epochs; such a change is considered a movement. The method used is nearly similar to the method described by van Hees et al. (2015). The epoch was classified as low movement, if the time window covering the preceding $10 \mathrm{~min}$ and following $10 \mathrm{~min}$ periods contained 0 or
1 movements; medium movement, if the window contained 2-13 movements; and high movement, if the window contained at least 14 movements. So, TIB indicates the whole time the accelerometer was worn on the wrist, and, within this period, the TIB was classified into three categories: low, medium, or high movement.

The participants were asked to keep a sleep diary for seven nights when they used the accelerometer at the wrist. The following data were gathered after waking up in the morning: (a) time gone to bed (hour; minutes), (b) number of times getting out of bed during sleeping, (c) wake-up time in the morning (hour; minutes), (d) perceived quality of sleep $(1=$ good to $5=$ poor $)$, and (e) perceived alertness after waking up $(1=$ very alert to $9=$ very sleepy).

\section{Assessment of Anthropometrics and CRF}

During the health examination, the trained research assistants measured participants' weight, height, and waist circumference. Weight and height were measured in light sporting clothes without shoes with a standardized digital scale and wall-mounted stadiometer. Body mass index was calculated as weight (in kilogram) divided by height (in meter square) and divided into three categories: $\leq 24.9 \mathrm{~kg} / \mathrm{m}^{2}, 25.0-29.9 \mathrm{~kg} / \mathrm{m}^{2}$, and $\geq 30.0 \mathrm{~kg} / \mathrm{m}^{2}$. Waist circumference (in centimeters) was measured on a horizontal plane halfway between the lower border of the ribs and the iliac crest. The mean of the three measurements was used as the test result.

The CRF was assessed by the 6-min walk test (American Thoracic Society, 2002). The research assistants asked participants to walk back and forth along the $15-\mathrm{m}$ walking track as fast as possible for $6 \mathrm{~min}$. Participants' heart rates were recorded with a heart rate monitor (Polar M61; Polar Electro, Kempele, Finland), and the distance walked was measured. For men, maximal oxygen consumption was estimated from the distance walked in $6 \mathrm{~min}$, age, body mass index, heart rate at the end of test, and height and, for women, from the distance walked in $6 \mathrm{~min}$, weight, and age. These predictors explained $82 \%$ of men's and $79 \%$ of women's measured maximal oxygen consumption corresponding to accuracy of about one MET. (Mänttäri et al., 2018). For the analysis, the test results were divided into fitness tertiles (low-fit, mid-fit, and high-fit) according to five age groups (20-29, 30-39, 40-49, 50-59, and 60-69 years) and sex.

\section{Background Characteristics}

As a part of the study questionnaire, participants were asked to report their educational background and marital status. Response options for educational background were (a) no vocational education, (b) vocational education, (c) Bachelor's degree, and (d) Master's degree. The response options for the marital status were (a) married, (b) cohabited, (c) single, (d) divorced, and (e) widowed.

\section{Statistical Methods}

Participants' characteristics are presented as means, $S D$, and percentages for categorical variables. Differences between men and women were tested with independent samples $t$ test (normally distributed continuous variables) and chi-square test (categorical variables). PA (light and MVPA), SB, standing, and TIB were analyzed in terms of total daily times according to age group and 
sex. Hour-by-hour patterns of SB, MVPA, and steps were analyzed according to age group and sex and patterns of steps and MVPA also according to CRF tertiles. Data are presented as mean with $S D$ or $95 \%$ confidence intervals. Differences in daily minutes between age groups and between sexes were analyzed with analysis of covariance. The $p$ values and confidence intervals were corrected for multiple comparisons by Sidak adjustment. Hourly differences were tested separately for each hour with Kruskal-Wallis $H$ test due to nonnormal distribution of the variables. The $p$ values $<.05$ were considered statistically significant. The analysis conducted according to weekdays and weekend days included only the participants who had accelerometer data for at least one weekend day. SPSS statistical software (IBM Statistics for Windows, Released 2019, version 26; IBM Corp., Armonk, NY) was used for all statistical analyses.

\section{Results}

Of the 5,723 persons out of 13,500 reached either by mail or phone, $2,378(41.6 \%)$ used the accelerometer at least to some extent. A total of 2,256 persons $(39.4 \%)$ wore the accelerometer at least 4 days for $24 \mathrm{hr} /$ day, which was the acceptance criterion for valid data collection. These eligible participants represented $95 \%$ of people who were basically willing to try the accelerometer. Most (78\%) of these eligible participants used the accelerometer for 6-7 days, and $97 \%$ of the participants had at least one weekend day in the data.

The mean age of the eligible participants $(N=2,256)$ was 49.5 years old (SD 13.5 years). Women comprised $59 \%$ of the participants (Table 1). Over half of the participants were married, and most of them had at least vocational education. On average, men were older $(p<.001)$ and had a higher body mass index $(p<.001)$, larger waist circumference $(p<.001)$, and higher CRF $(p<.001)$ than the women. Also, marital status $(p<.001)$ and level of education $(p=.002)$ differed between the sexes.

The Figure 1 shows a sample of one measurement day. When awake, the frequency of the sensor movements was higher, but the proportion of the large movements was smaller than during TIB. Participants wore the accelerometer in the hip belt, on average, $15 \mathrm{hr} 40 \mathrm{~min} /$ day and, in the wrist band (TIB), $8 \mathrm{hr} 20 \mathrm{~min} /$ day (SD $1 \mathrm{hr} 28 \mathrm{~min}$ ). According to the sleep diary, the mean sleeping time was $8 \mathrm{hr} 2 \mathrm{~min} /$ day ( $S D 53 \mathrm{~min}$ ), indicating that the TIB measured by the accelerometer was $17.7 \mathrm{~min}$ (SD $1 \mathrm{hr} 24 \mathrm{~min}$ ) longer than reported sleeping time on average.

Figure 2 presents the distribution of different behaviors (TIB [accelerometer at the wrist] and SB, standing, light PA, moderate PA, and vigorous PA [accelerometer at the hip]) during these hours according to age group and sex. Supplementary Table 1 (available online) shows the $95 \%$ confidence intervals and SDs for the mean values.

\section{Physical Activity}

Participants had, on average, $3 \mathrm{hr} 42 \mathrm{~min}$ of light PA $(S D=1 \mathrm{hr}$ $16 \mathrm{~min}$ ) per day (women $3 \mathrm{hr} 45 \mathrm{~min}$ and men $3 \mathrm{hr} 37 \mathrm{~min}, p=.02$ ) (Figure 2). The youngest ( $3 \mathrm{hr} 28 \mathrm{~min}$ ) and the oldest ( $3 \mathrm{hr} 26 \mathrm{~min}$ ) age groups had, on average, less light PA than the other age groups (from $p=.01$ to $p<.001$ ). The amount of light PA of the other age groups did not differ from each other.

The mean daily time spent in MVPA was $46 \min (S D=$ $26 \mathrm{~min})$. Men had, on average, 3 min more daily MVPA than women (48 min vs. $45 \mathrm{~min}, p=.006$ ). The oldest age group had, on average, less MVPA (41 min) than the other age groups ( $p<$ $.001)$. The youngest participants had on average the highest time of daily MVPA (52min), but their MVPA did not differ statistically significantly from that of the $30-39$ or $40-49$ year old participants.

The mean daily step number was 7,451 ( $S D=2,962$ steps). There was no difference between the sexes (see Supplementary Figure 1 [available online]), but the oldest age group took, on average, 1,000 less steps daily than all the younger groups $(p<.001)$

\section{Hour-by-Hour Patterns of PA}

Figure 3 presents the hour-by-hour patterns of daily steps and MVPA in age groups. Among both men and women, the oldest participants accumulated the most steps and most MVPA minutes per hour at noon, and the hourly step number and MVPA minutes decreased in the afternoon and evening. The women aged younger than 60 years had two peaks in the daily step number and MVPA minutes, one at noon and the other one in the evening, around 6 p.m. The hour-by-hour differences between the age groups are presented in Supplementary Table 2 (available online).

When the hour-by-hour patterns of PA were assessed in sexand age-specific tertiles of CRF, the low-fit tertile accumulated the least PA assessed, both in terms of step number and MVPA minutes (Figure 4). The low-fit tertile took, on average, 1,186 fewer steps during the day than the mid-fit tertile $(p<.001)$ and 1,747 fewer steps than the high-fit tertile $(p<.001)$. Correspondingly, the low-fit tertile had, on average, $9.8 \mathrm{~min}$ less MVPA during the day than the mid-fit tertile $(p<.001)$ and 15.0 min less than the high-fit tertile $(p<.001)$. The high-fit tertile accumulated the highest hour-by-hour step number and MVPA minutes at noon and at the evening hours. Among women, the two-peak pattern in steps and MVPA minutes was more distinct compared with men, and this pattern also occurred among the mid-fit tertile. Supplementary Table 3 (available online) presents the hour-by-hour differences between the age groups.

The hour-by-hour patterns of steps and MVPA minutes differed between weekdays and weekend days among both men and women (see Supplementary Figures 2 and 3 [available online]). The participants in the low-fit tertile accumulated the least number of steps and MVPA minutes during both weekdays and weekend days. In general, participants accumulated, on average, more steps and MVPA minutes during weekdays than during weekend days both in the mornings and the evening hours, whereas, during the weekend days, the hours around noon were, on average, more active than the ones during weekdays.

Both men and women in the high-fit tertile reached the highest hour-by-hour step number and MVPA minutes during the weekend days, around the noon. In the afternoon, the hour-by-hour PA decreased, showing a small peak around 6 p.m. among the men. During weekdays, the highest step number was reached during evening hours, and high-fit women accumulated, on average, higher hour-by-hour steps and MVPA minutes than high-fit men. The difference between the fitness tertiles on weekdays was statistically significant from 7 a.m. to 12 p.m. $(p<.05)$, regarding the steps, and from 6 a.m. to 12 p.m. $(p<.05)$, regarding the MVPA minutes. On weekends, the corresponding differences were statistically significant from 8 a.m. to 12 p.m. $(p<.05)$, regarding both steps, and MVPA minutes, except for MVPA from 9 p.m. to 10 p.m. 
Table 1 General Characteristics of the Participants $(N=2,256)$, Mean Values With $S D$, or Percentage Distributions With $p$ Value for the Difference Between Men and Women

\begin{tabular}{|c|c|c|c|}
\hline Variable & Men & Women & $p$ value \\
\hline \multicolumn{4}{|l|}{ Age } \\
\hline Mean $(S D)$ & $50.7(13.2)$ & $48.6(13.6)$ & $<.001^{\mathrm{a}}$ \\
\hline$n$ & 918 & 1,338 & \\
\hline \multicolumn{4}{|l|}{ Age group (\%) } \\
\hline $20-29$ & 7.6 & 11.0 & $.013^{\mathrm{b}}$ \\
\hline $30-39$ & 16.1 & 17.0 & \\
\hline $40-49$ & 19.2 & 20.7 & \\
\hline $50-59$ & 24.1 & 23.8 & \\
\hline $60-69$ & 33.0 & 27.6 & \\
\hline$n$ & 918 & 1,338 & \\
\hline \multicolumn{4}{|l|}{ Weight (kg) } \\
\hline Mean $(S D)$ & $86(14)$ & $70(14)$ & $<.001^{\mathrm{a}}$ \\
\hline$n$ & 831 & 1,144 & \\
\hline \multicolumn{4}{|l|}{ Height (cm) } \\
\hline Mean $(S D)$ & $178(11)$ & $165(9)$ & $<.001^{\mathrm{a}}$ \\
\hline$n$ & 831 & 1,143 & \\
\hline \multicolumn{4}{|l|}{ BMI $\left(\mathrm{kg} / \mathrm{m}^{2}\right)$} \\
\hline Mean $(S D)$ & $26.9(4.0)$ & $25.8(4.8)$ & $<.001^{\mathrm{a}}$ \\
\hline$n$ & 829 & 1,141 & \\
\hline \multicolumn{4}{|l|}{ BMI (\%) } \\
\hline$<24.9$ & 67.3 & 74.8 & $<.001^{\mathrm{a}}$ \\
\hline $25.0-29.9$ & 13.6 & 7.4 & \\
\hline$\geq 30.0$ & 19.1 & 17.8 & \\
\hline$n$ & 829 & 1,141 & \\
\hline \multicolumn{4}{|l|}{ Waist circumference $(\mathrm{cm})$} \\
\hline Mean $(S D)$ & $96.8(12.8)$ & $87.3(14.0)$ & $<.001^{\mathrm{a}}$ \\
\hline$n$ & 831 & 1,143 & \\
\hline \multicolumn{4}{|l|}{ Marital status (\%) } \\
\hline Married & 60.1 & 50.2 & $<.001^{\mathrm{b}}$ \\
\hline Cohabited & 21.1 & 21.0 & \\
\hline Single & 10.6 & 15.3 & \\
\hline Divorced & 7.5 & 11.0 & \\
\hline Widowed & 0.6 & 2.6 & \\
\hline$n$ & 810 & 1,212 & \\
\hline \multicolumn{4}{|l|}{ Education (\%) } \\
\hline No vocational education & 13.7 & 8.9 & $.002^{\mathrm{b}}$ \\
\hline Vocational education & 45.9 & 44.6 & \\
\hline Bachelor's degree & 18.4 & 21.8 & \\
\hline Master's degree & 21.9 & 24.7 & \\
\hline$n$ & 808 & 1,212 & \\
\hline \multicolumn{4}{|c|}{ Cardiorespiratory fitness, $\mathrm{VO}_{2}$ peak, $\left(\mathrm{ml} \cdot \mathrm{kg}^{-1} \cdot \mathrm{min}^{-1}\right)$} \\
\hline Mean $(S D)$ & $35.5(6.7)$ & $31.8(6.4)$ & $<.001^{\mathrm{a}}$ \\
\hline$n$ & 778 & 1,101 & \\
\hline \multicolumn{4}{|c|}{ Accelerometer use: number of 24-hr days } \\
\hline Mean $(S D)$ & $5.8(0.8)$ & $5.9(0.7)$ & $.031^{\mathrm{a}}$ \\
\hline$n$ & 918 & 1,338 & \\
\hline
\end{tabular}

Note. $\mathrm{BMI}=$ body mass index $; \mathrm{VO}_{2}$ peak $=$ maximal oxygen consumption .

${ }^{\mathrm{a}}$ Independent samples $t$ test. ${ }^{\mathrm{b}} \mathrm{Chi}$-square test. 
(a)

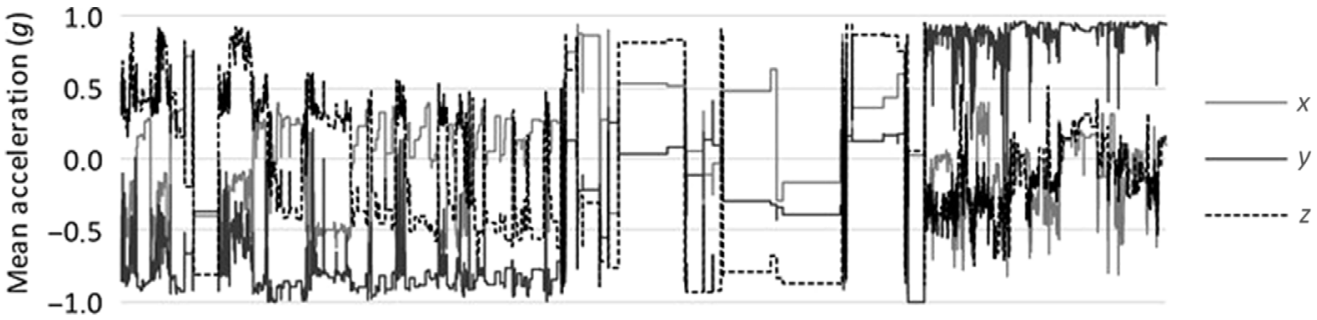

(b)

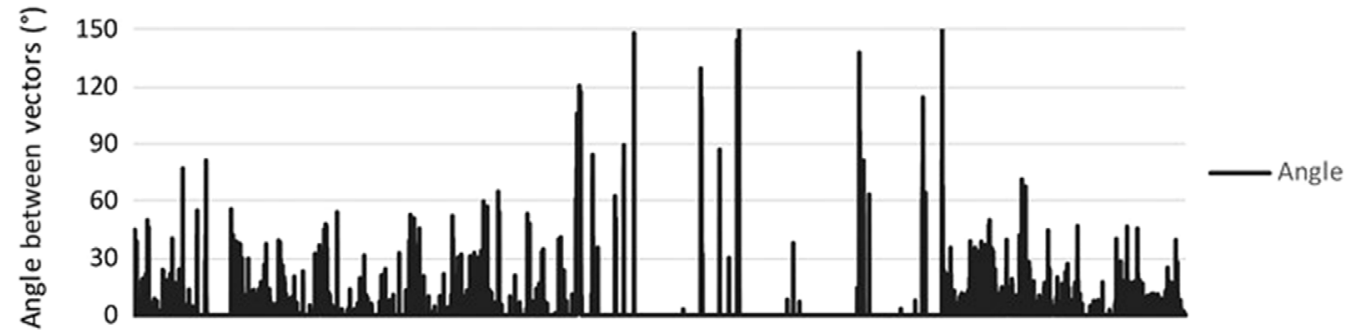

(c)

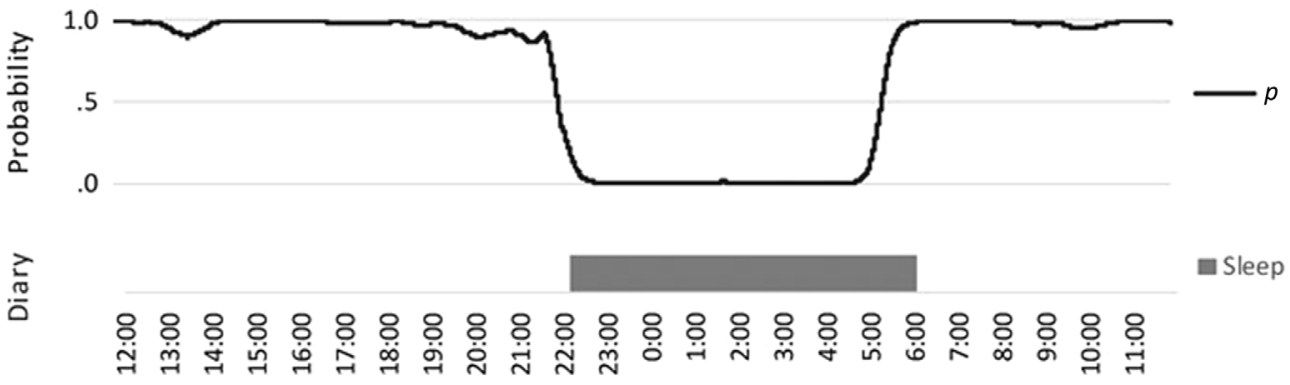

Figure 1 - Graphics of the differentiating sensor location method: (a) the epoch-wise measurement axis specific mean values, (b) the angle between adjacent epochs, (c) the output of the binary logistic regression model and (d) the sleep time according to the diaries.

\section{SB and Standing}

Mean SB time was $9 \mathrm{hr} 18 \mathrm{~min}(S D=1 \mathrm{hr} 48 \mathrm{~min})$ in all age and sex groups (Figure 2). The mean sedentary time was higher in men than in women $(9 \mathrm{hr} 44 \mathrm{~min}$ vs. $9 \mathrm{hr} 1 \mathrm{~min}, p<.001)$. The oldest age group ( $9 \mathrm{hr} 44 \mathrm{~min}$ ) had, on average, higher SB time per day than the 30-39, 40-49, and 50-59 year old groups $(p<.001)$. Also, the youngest age group had statistically significantly more SB $(9 \mathrm{hr}$ $25 \mathrm{~min})$ than the 30- to 39-year-old $(p=.007)$ and 40- to 49-yearold $(p=.026)$ participants.

Participants' mean daily standing time was $1 \mathrm{hr} 54 \mathrm{~min}$ $(S D=55 \mathrm{~min})$. Women stood, on average, more than men $(2 \mathrm{hr}$ $4 \mathrm{~min}$ vs. $1 \mathrm{hr} 40 \mathrm{~min}, p<.001)$. The youngest (1 hr $42 \mathrm{~min}$ ) and oldest ( $1 \mathrm{hr} 45 \mathrm{~min}$ ) age groups stood statistically significantly less time per day than the other age groups (from $p=.009$ to $p<.001$ ).

\section{Hour-by-Hour Pattern of SB}

Hourly sedentary minutes increased slightly toward the evenings, especially among the oldest participants (Figure 5). Age grouprelated differences in hour-by-hour pattern of sedentary minutes are presented in Supplementary Table 2 (available online).

\section{Time in Bed}

The mean TIB was 14 min longer among women than among men ( 8 hr 11 min vs. 8 hr 25 min, $p<.001$; Figure 2). The youngest age group (20- to 29-year-olds) had the longest TIB (8 hr $33 \mathrm{~min}$ ) and 40to 49 -year-old participants the shortest one $(8 \mathrm{hr} 5 \mathrm{~min})(p=.002)$.

When TIB was assessed in terms of low, medium, and high movement times, women had, on average, 21 min longer low movement time than men $(p<.001)$, but medium movement time did not differ between sexes. Younger age groups had, on average, shorter low movement time than the older ones: The 20- to 29year-old participants differed significantly from those aged 30 years and older $(p=.006)$, and the 30- to 39-year-old and 40- to 49-year-old participants differed from those aged 50 years and older $(p=.002)$. The 60- to 69-year-old participants had the highest amount of low movement time. Regarding medium movement time, there was no difference between the sexes. The youngest participants had the highest amount of medium movement time, which declined gradually toward the older age groups. The 20- to 29-year-old participants differed statistically significantly from those aged 40 years and older $(p<.001)$. Also, the $30-$ to 39-year-old participants differed from those aged 40-49 years $(p=.024)$ and from those aged 50 years and older $(p<.001)$, and the 40- to 49-year-old participants differed from the oldest age group $(p=.007)$. Men had, on average, 4 min more high movement time during the TIB than the women $(p=.030)$. The youngest age group had, on average, the longest high movement time and the 40- to 49-years-old participants had the shortest time. The difference between these two age groups was statistically significant $(p<.001)$. The youngest group differed also from the 50- to 59 -year-old participants $(p=.004)$ and the 40- to 49-year-old 


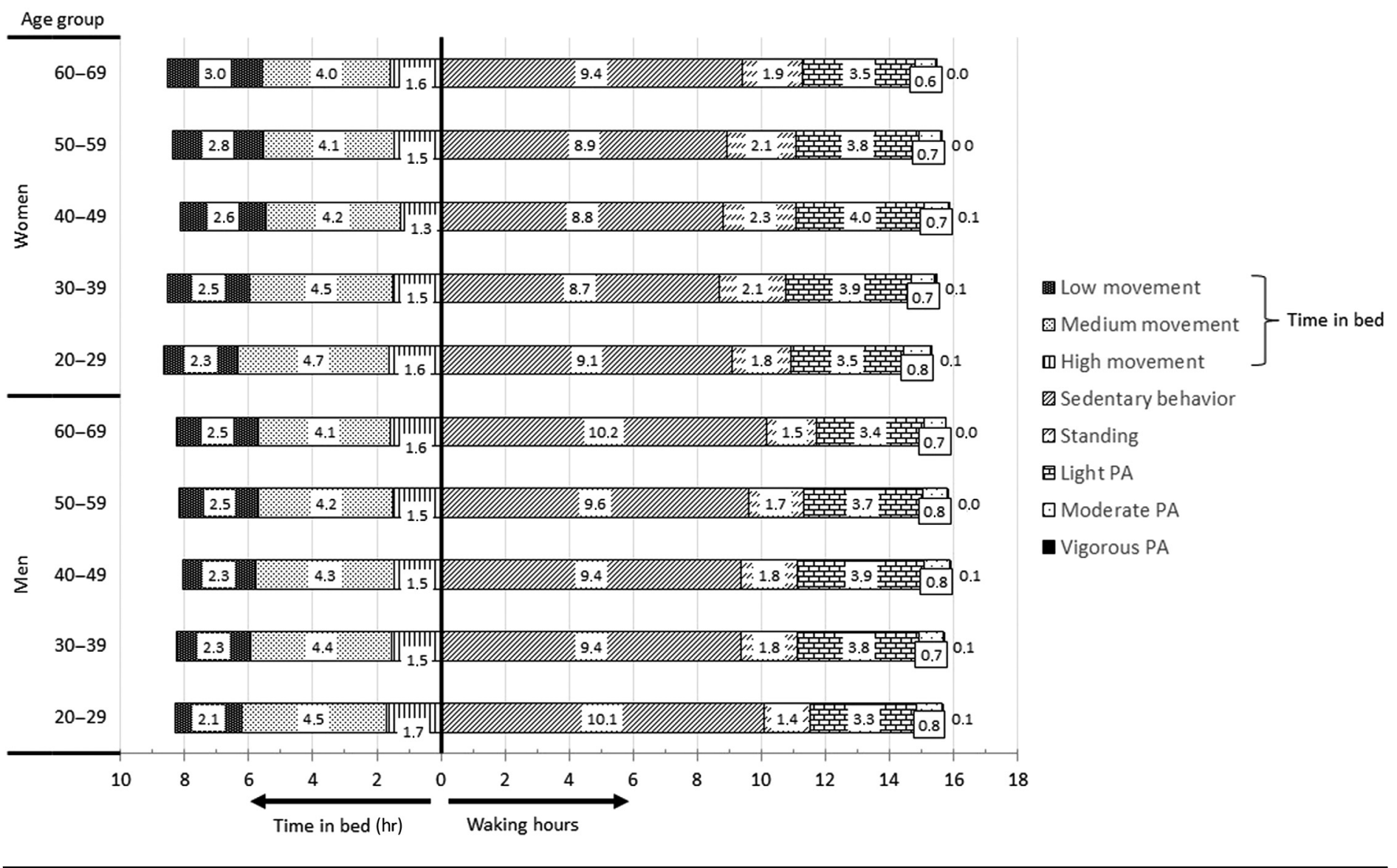

Figure 2 - Distribution of different behaviors: time in bed; sedentary behavior; standing; and light, moderate and vigorous physical activity during a day. During time in bed the accelerometer was worn on the wrist band and during waking hours on the hip belt. PA=physical activity.

participants differed from the 60- to 69-year-old $(p<.001)$ and the 30- to 39-year-old participants $(p=.007)$.

\section{Discussion}

The present population-based study described sex- and agegroup differences in daily PA, SB, standing, and TIB among Finnish working-aged adults during a 24/7 measurement period. Furthermore, the study presented hour-by-hour patterns of SB, steps, and MVPA. To our knowledge, this is the first time that these physical behaviors have been measured 24/7 in a population-based sample of Finnish adults using both hip- and wristworn triaxial accelerometry collecting data in raw mode. The unique feature of the present study was that the device was worn at the hip during waking hours and at the wrist during TIB. Because of relocating the accelerometer, we were able to identify when the participants intended to go to sleep and when they perceived they had woken up. The comparison of the accelerometer data and sleeping diary showed that the accelerometermeasured TIB was nearly 18 min longer on average. Also, the variation of the TIB was larger according to accelerometer data ( $S D$ for TIB $1 \mathrm{hr} 28$ min vs. $S D$ for sleeping time from the diary $53 \mathrm{~min}$ ). The participants may have filled in the diary for several days at a time, which may be prone to recall bias. This may have equalized the true bed-in and bed-out times, decreasing the reliability of self-reported data.
The findings of the present study showed that $95 \%$ of those who were willing to try the accelerometer met the criterion for acceptable accelerometer use (at least 4 days, with $24 \mathrm{hr}$ of measurement per day): $78 \%$ of them used the device for 6 7 days, and $97 \%$ had at least one weekend day in the data, which indicates that the two wear-site approach used in the study was feasible among the working-aged adults.

The novel findings of the study indicated that the most active hours in men occurred at noon and, those in women, at noon and at early evening. This two-peak fashion of PA among women aged 20-59 year is in line with previous findings (Wennman et al., 2019). Also in line with previous findings (Willetts, Hollowell, Aslett, Holmes, \& Doherty, 2018, Wennman et al., 2019), older participants in the present study were less active than the younger ones, and they reached their highest PA levels earlier in the day.

Participants of the present study spent daily over $9 \mathrm{hr}$ of their waking hours either sitting or lying down on average. Standing comprised nearly $2 \mathrm{hr} /$ day, light PA over $3.5 \mathrm{hr}$, and MVPA $<1 \mathrm{hr} /$ day. Men had, on average, more MVPA and SB than women, while the total standing time in women was, on average, longer than in men. In the present study, women had, on average, 8 min more light PA than men. The sex differences of the present study regarding $\mathrm{SB}$, light PA, and MVPA are in line with the wrist-based measurements conducted among older adults (Cabanas-Sánchez et al., 2020). Wrist-based measurements by Willetts et al. (2018), on the other hand, indicated that men spent more time in low-intensity activities, like sitting, and more time in purposeful PA, like 
Steps per hour

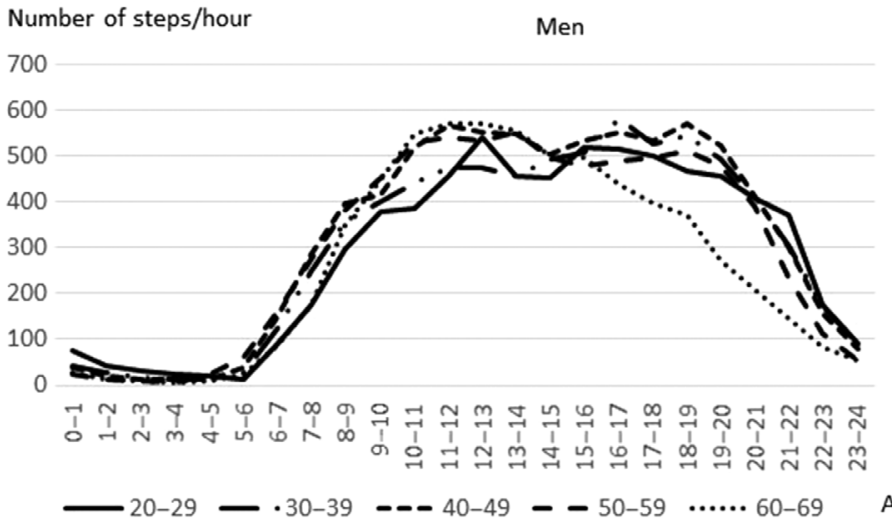

MVPA per hour

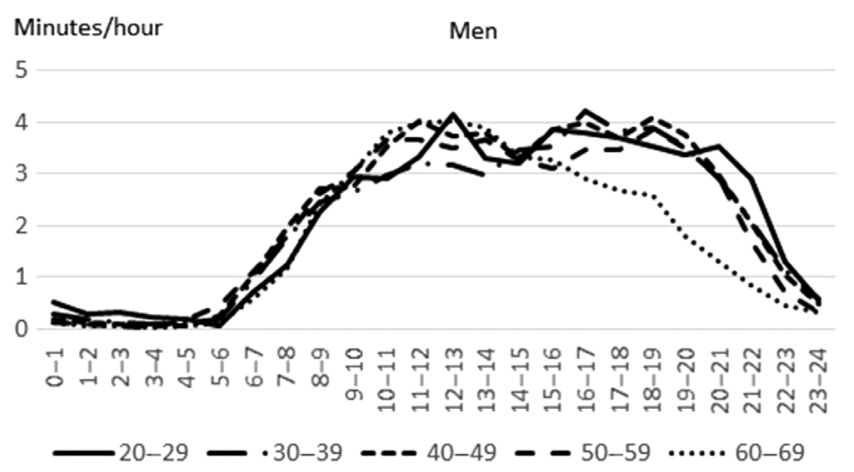

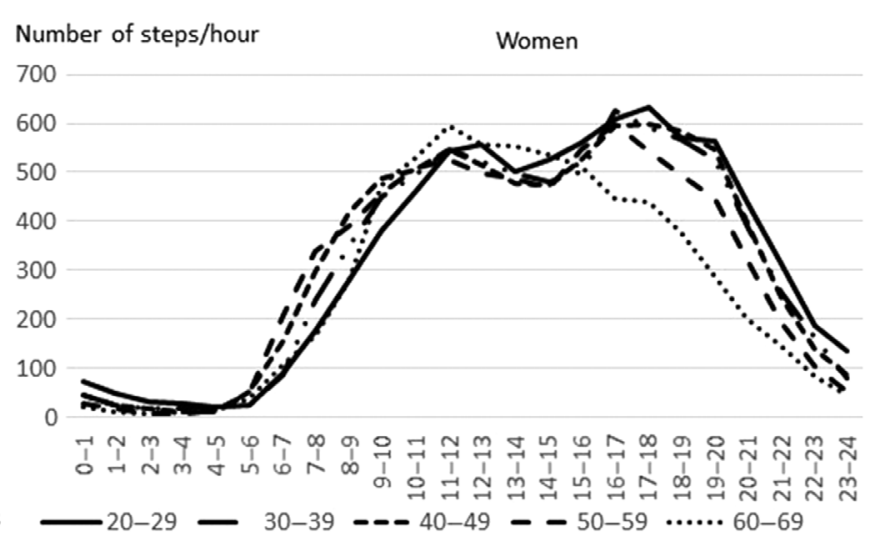

Age groups

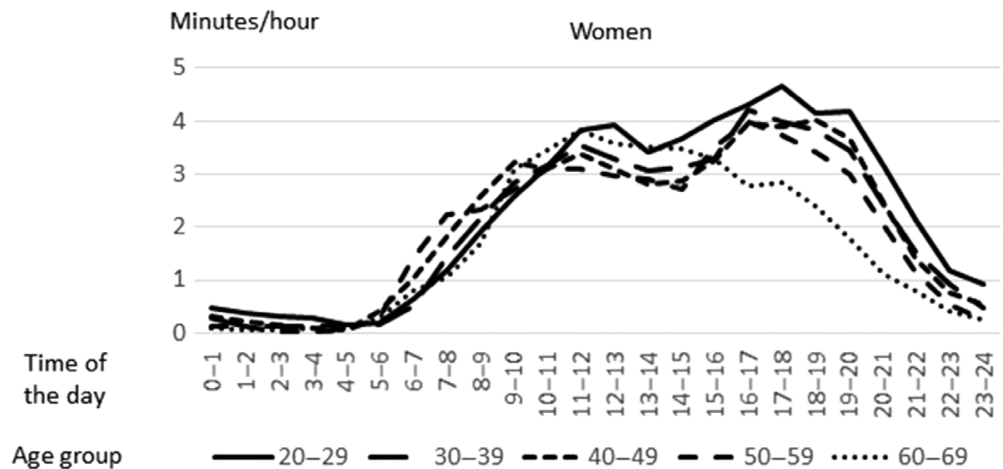

Figure 3 - Hour-by-hour patterns of daily steps and MVPA in age groups and sex categories. MVPA=moderate to vigorous PA; PA=physical activity.

walking, than women. A recent Finnish study based on wrist measurements reported that women accumulated more PA in general during the day than men (Wennman et al., 2019). The difference between the present findings and those of Willetts et al. (2018) and Wennman et al (2019) may be partly due to device placement as hip- and wrist-worn devices seem to capture somewhat different behaviors during waking hours (Leinonen et al., 2017, Shiroma et al., 2016).

Besides the comprehensive 24/7 SB, PA, and TIB measurements, another strength of the present study was the assessment of CRF. The PA pattern of the low-fit tertile differed significantly from that of the high-fit tertile when the step number and MVPA minutes were used as indicators of PA. In addition, the low-fit tertile was less active than the high-fit tertile during all waking hours. The step number of the present study included all steps irrespective of stepping intensity or cadence. A previous study (Kujala et al., 2017) showed that low-fit individuals are physically less active than the fittest ones, mainly due to their inability to reach vigorous PA levels in absolute terms, but when PA intensity is estimated relative to individual fitness levels, the differences are much smaller. The present study showed very similar PA patterns for both steps and MVPA, most of which consisted of moderateintensity activity.

To our knowledge, the present study is the first one that has described the differences in daily PA patterns according to fitness level, also by comparing weekdays and weekend days. The hourby-hour patterns of PA in the low-fit tertile did not show any peaks during the day, neither on weekdays nor weekend days, while the
PA patterns of the high-fit tertile showed a clear peak at noon on the weekend days. Our results present the step and MVPA patterns of adult participants; however, we were not able to analyze the patterns according to context of activity nor the working status and/or type of work of the participants. In future studies, it would be important to also analyze hourly PA patterns in the proper context.

Regarding TIB, the women had, on average, more low movement time nightly than the men, while there was no difference between the sexes in the amount of medium movement time. The mean low movement time increased slightly from younger to older age groups, while the mean medium movement time increased toward younger groups. Assessing TIB (a proxy for sleeping) by wrist-worn accelerometers, Willetts et al. (2018) reported that older participants spent a greater proportion of measurement time sleeping than the younger ones. So far, the studies have used different devices, different device placements, and different algorithms to identify sleep and sleep-related behavior, which makes the comparisons between studies very challenging. Gibbs and Kline (2018) proposed that although sleep-related behaviors, like time spent trying to fall asleep, night awakenings while attempting to fall back asleep, and unsuccessful attempts to fall back asleep in the morning, fulfill the criteria for SB (Tremblay et al., 2017), they are part of the normal and healthy sleep rhythm and thus not targets for SB reduction. Further population-based studies with more reliable sleep assessments are needed for gaining insight into these sleep-related behaviors. The golden standard of sleep research, polysomnography, is far beyond what is feasible in populationbased studies. 
Steps per hour
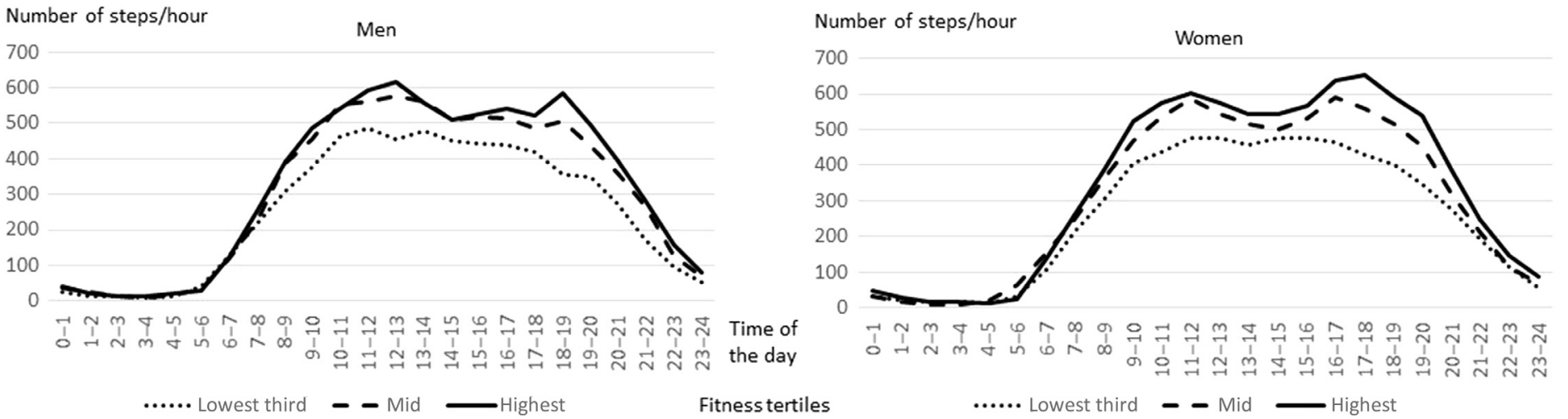

MVPA per hour
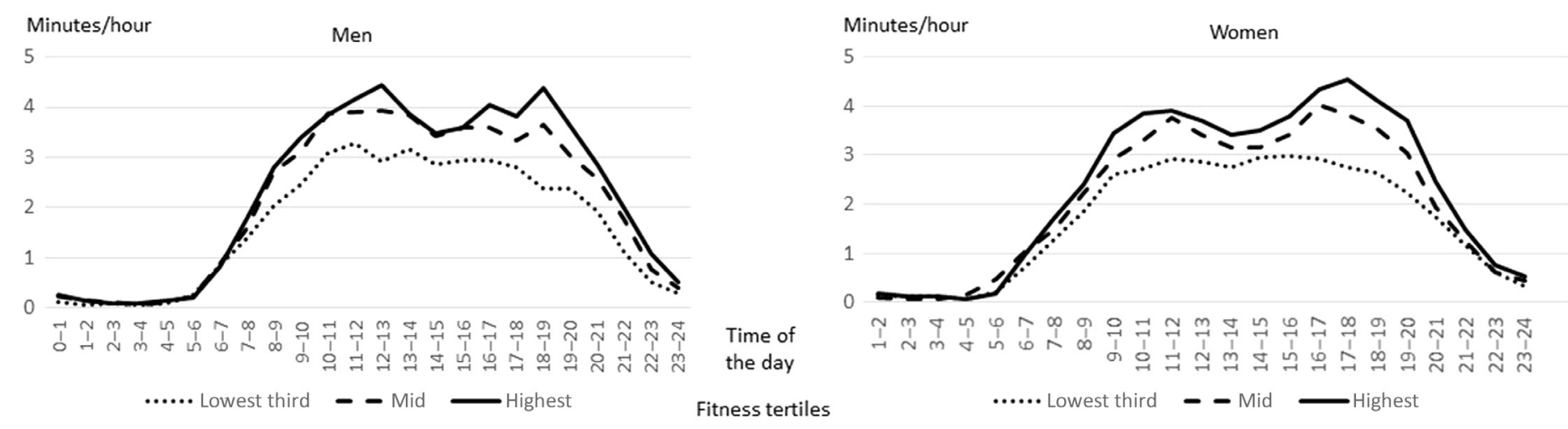

Figure 4 - Hour-by-hour patterns of steps and MVPA according to the cardiorespiratory fitness tertiles. MVPA=moderate to vigorous PA; $\mathrm{PA}=$ physical activity.

\section{Sedentary minutes per hour}

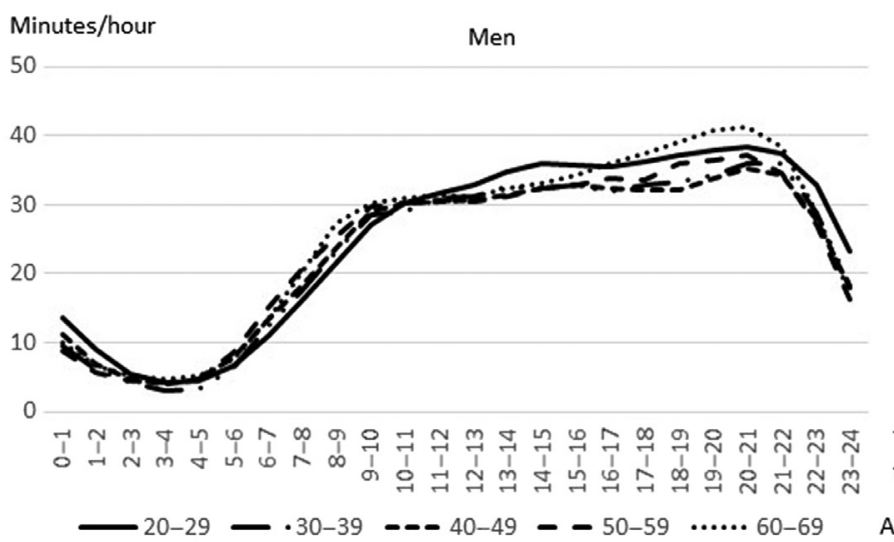

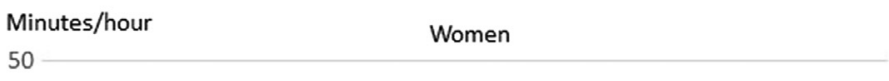

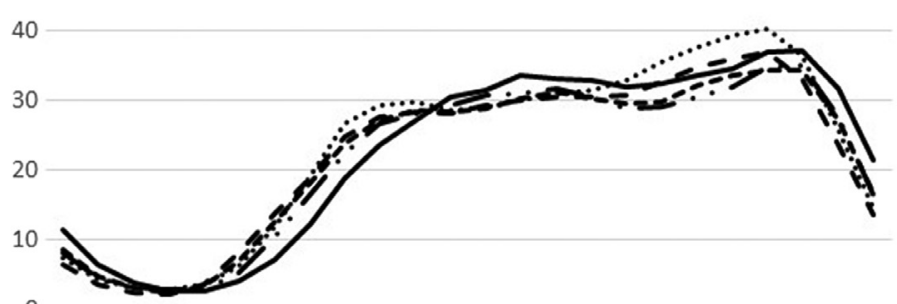

Time of 구 1)

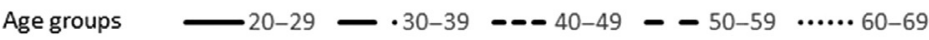

Figure 5 - Hour-by-hour patterns of sedentary behavior in age groups and sex categories.

The strengths of the present study were the 24/7 measurement of different behaviors (low, medium, and high movements during TIB and SB, standing, light PA, and MVPA during waking hours); use of two different sites for accelerometer, depending on the purpose of the measurement; population-based sample of seven urban and suburban areas covering a wide age range from 20 to 69 years; and evaluation of CRF. The main weakness of the study was the selective participation, where only $42 \%$ of the sample was reached, and $51 \%$ of the reached persons agreed to participate in the study. The study participants were more likely to be married (Official Statistics of Finland, 2020) and were more educated than the general population in Finland (Official Statistics of Finland, 2018), which may have affected the present findings. 


\section{Conclusions}

The findings of the present study help to draw the whole picture of PA, SB, and TIB patterns among working-aged Finnish adults. The findings confirm that the present method of utilizing one triaxial accelerometer with a two wear-site approach is feasible to assess physical behaviors $24 / 7$ in working-aged adults. Furthermore, assessment of PA according to fitness tertiles provides descriptive information of the differences in PA patterns between fitness levels. In future population-based studies, the present method can be utilized to assess health-related associations of comprehensive 24/7 device-measured PA, SB, and TIB.

\section{Acknowledgments}

Laboratory staff of the participating research centers, Santasport Rovaniemi, City of Helsinki, and Turku University of Applied Sciences are acknowledged for their help in the data collection. Lifestyles of Health and Sustainability to Boost Sustainable Growth Consortium, Academy of Finland, Strategic Research Council is acknowledged for collaboration (320400 and 320403). The study was financially supported by the Finnish Ministry of Education and Culture.

\section{References}

American Thoracic Society. (2002). ATS statement: Guidelines for the sixminute walk test. American Journal of Respiratory and Critical Care Medicine, 166, 111-117. doi:10.1164/ajrccm.166.1.at1102

Barreira, T.V., Zderic, T.W., Schuna, J.M., Jr., Hamilton, M.T, \& TudorLocke, C. (2015). Free-living activity counts-derived breaks in sedentary time: Are they real transitions from sitting to standing? Gait \& Posture, 42(1), 70-72. PubMed ID: 25953504 doi:10.1016/j. gaitpost.2015.04.008

Bassett, D.R., Jr., Toth, L.P., LaMunion, S.R., \& Crouter, S.E. (2017). Step counting: A review of measurement considerations and health-related applications. Sports Medicine, 47(7), 1303-1315. PubMed ID: 28005190 doi:10.1007/s40279-016-0663-1

Cabanas-Sánchez, V., Esteban-Cornejo, I., Migueles, J.H., Banegas, J.R., Graciani, A., Rodríguez-Artalejo, F., \& Martínez-Gómez, D. (2020). Twenty four-hour activity cycle in older adults using wrist-worn accelerometers: The seniors-ENRICA-2 study. Scandinavian Journal of Medicine and Science in Sports, 30(4), 700-708. PubMed ID: 31834945 doi:10.1111/sms.13612

Caramaliu, R.V., Vasile, A., \& Bacis, I. (2015). Wearable vital parameters monitoring system. Advanced Topics in Optoelectronics, Microelectronics, and Nanotechnologies VII, 9258, 92580R. doi:10.1117/12. 2070041

Chaput, J.P., Carson, V., Gray, C.E., \& Tremblay, M.S. (2014). Importance of all movement behaviors in a 24 hour period for overall health. International Journal of Environmental Research and Public Health, 11(12), 12575-12581. PubMed ID: 25485978 doi:10.3390/ ijerph111212575

Chaudhry, U.M.R., Wahlich, C., Fortescue, R., Cook, D.G., Knightly, R., \& Harris, T. (2020). The effects of step-count monitoring interventions on physical activity: Systematic review and meta-analysis of community based randomised controlled trials in adults. International Journal of Behavioral Nutrition and Physical Activity, 17(1), 129. doi:10.1186/s12966-020-01020-8

Crowley, P., Skotte, J., Stamatakis, E., Hamer, M., Aadahl, M., Stevens, M.L., ... Holtermann, A. (2019). Comparison of physical behavior estimates from three different thigh-worn accelerometer brands: A proof-of-concept for the prospective physical activity, sitting and sleep consortium (ProPASS). International Journal of Behavioral Nutrition and Physical Activity, 16, 65. doi:10.1186/s12966-019-0835-0

Doherty, A., Jackson, D., Hammerla, N., Plötz, T., Olivier, P., Granat, M.H., .. Wareham, N.J. (2017). Large scale population assessment of physical activity using wrist worn accelerometers: The UK Biobank Study. PLoS One, 12(2), e0169649. doi:10.1371/journal.pone. 0169649

Gibbs, B.B., \& Kline, C.E. (2018). When does sedentary behavior become sleep? A proposed framework for classifying activity during sleep-wake transitions. International Journal of Behavioral Nutrition and Physical Activity, 15(1), 81. doi:10.1186/s12966018-0712-2

Hees, V.T., Sabia, S., Anderson, K.N., Denton, S.J., Oliver, J., Catt, M., ... Singh-Manoux, A. (2015). A novel, open access method to assess sleep duration using a wrist-worn accelerometer. PLoS One, 10(11), e0142533. PubMed ID: 26569414 doi:10.1371/journal.pone. 0142533

Husu, P., Suni, J., Vähä-Ypyä, H., Sievänen, H., Tokola, K., Valkeinen, H., . . Vasankari T. (2016). Objectively measured sedentary behavior and physical activity in a sample of Finnish adults: A crosssectional study. BMC Public Health, 16(1), 920. PubMed ID: 27586887 doi:10.1186/s12889-016-3591-y

Jaeschke, L., Luzak, A., Steinbrecher, A., Jeran, S., Ferland, M., Linkohr, B., ... Pischon, T. (2017). 24 h-Accelerometry in epidemiological studies: Automated detection of non-wear time in comparison to diary information. Scientific Reports, 7(1), 2227. PubMed ID: 28533553 doi:10.1038/s41598-017-01092-w

Judice, P.B., Santos, D.A., Hamilton, M.T., Sardinha, L.B., \& Silva, A.M. (2015). Validity of GT3X and actiheart to estimate sedentary time and breaks using ActivPAL as the reference in free-living conditions. Gait \& Posture, 41(4), 917-922. PubMed ID: 25852024 doi:10.1016/j. gaitpost.2015.03.326

Koolhaas, C.M., van Rooij, F.J.A., Schoufour, J.D., Cepeda, M., Tiemeier, H., Brage, S., \& Franco, O.H. (2017). Objective measures of activity in the elderly: Distribution and associations with demographic and health factors. Journal of the American Medical Directors Association, 18(10), 838-847. PubMed ID: 28602617 doi:10.1016/j.jamda. 2017.04.017

Kujala, U.M., Pietilä, J., Myllymäki, T., Mutikainen, S., Föhr, T., Korhonen, I., \& Helander, E. (2017). Physical Activity: Absolute intensity versus relative-to-fitness-level volumes. Medicine and Science in Sports and Exercise, 49(3), 474-481. PubMed ID: 27875497 doi:10.1249/MSS.0000000000001134

Lee, D-C., Sui, X., Ortega, F.B., Kim, Y-S., Church, T.S., Winett, R.A., ... Blair, S.N. (2011). Comparisons of leisure-time physical activity and cardiorespiratory fitness as predictors of all-cause mortality in men and women. British Journal of Sports Medicine, 45(6), 504-510. PubMed ID: 20418526 doi:10.1136/bjsm.2009.066209

Leinonen, A.M., Ahola, R., Kulmala, J, Hakonen, H., Vähä-Ypyä, H., Herzig, K-H., ... Jämsä, T. (2017). Measuring physical activity in free-living conditions-comparison of three accelerometry-based methods. Frontiers in Physiology, 10(7), 681. doi:10.3389/fphys. 2016.00681

Lyden, K., Kozey-Keadle, S., Staudenmayer, J., Freedson, P.S. (2017). The activPAL accurately classifies activity intensity categories in healthy adults. Medicine and Science in Sports and Exercise, 49(5), 1022-1028. PubMed ID: 28410327 doi:10.1249/MSS.00000000 00001177

Mänttäri, A., Suni, J., Sievänen, H., Husu, P., Vähä-Ypyä, H., Valkeinen, H., ... Vasankari, T. (2018). Six-minute walk test: a tool for predicting maximal aerobic power $\left(\mathrm{VO}_{2} \mathrm{max}\right)$ in healthy adults. 
Clinical Physiology and Functional Imaging, 38(6), 1038-1045. doi:10.1111/cpf.12525

Niemelä, M., Kangas, M., Farrahi, V., Kiviniemi, A., Leinonen, A-M., Ahola, R., ... Jämsä, T. (2019). Intensity and temporal patterns of physical activity and cardiovascular disease risk in midlife. Preventive Medicine, 124, 33-41. doi:10.1016/j.ypmed.2019.04.023

Official Statistics of Finland (OSF). (2018). Educational structure of population [e-publication]. Helsinki: Statistics Finland. ISSN 2242-2919. Retrieved from http://www.stat.fi/til/vkour/2017/vkour_ 2017_2018-11-02_tie_001_en.html

Official Statistics of Finland (OSF). (2020). Population structure [e-publication]. Helsinki: Statistics Finland. ISSN 1797-5395. Retrieved from http://www.stat.fi/til/vaerak/index_en.html

Pedisic, Z., Dumuid, D., \& Olds, T.S. (2017). Integrating sleep, sedentary behavior, and physical activity research in the emerging field of timeuse epidemiology: Definitions, concepts, statistical methods, theoretical framework and future directions. Kinesiology, 49(2), 252-269.

Ramires, V.V., Wehrmeister, F.C., Wendt Böhm, A., Galliano, L., Ekelund, U., Brage, S., \& da Silva, I.C.M. (2017). Physical activity levels objectively measured among older adults: a population-based study in a Southern city of Brazil. International Journal of Behavioral Nutrition and Physical Activity, 14(1), 13. doi:10.1186/s12966-017-0465-3

Rosenberger, M.E., Fulton, J.E., Buman, M.P., Troiano, R.P., Grandner, M.A., Buchner, D.M., \& Haskell, W.L. (2019). The 24-hour activity cycle: A new paradigm for physical activity. Medicine and Science in Sports and Exercise, 51(3), 454-464. PubMed ID: 30339658 doi:10. 1249/MSS.0000000000001811

Rosenberger, M.E., Haskell, W.L., Albinali, F., Mota, S., Nawyn, J., \& Intille, S. (2013). Estimating activity and sedentary behavior from an accelerometer on the hip or wrist. Medicine and Science in Sports and Exercise, 45(5), 964-975. PubMed ID: 23247702 doi:10.1249/MSS. 0b013e31827f0d9c

Saint-Maurice, P.F., Troiano, R.P., Basset, D.R., Jr., Graubard, B.I., Carlson, S.A, Shiroma, E.J, ... Matthews, C.E. (2020). Association of daily step count and step intensity with mortality among US adults. JAMA, 323(12), 1151-1160. PubMed ID: 32207799 doi:10.1001/ jama.2020.1382

Schram, M.T., Sep, S.J., van der Kallen, C.J., Dagnelie, P.C., Koster, A., Schaper, N., ... Stehouwer, C.D. (2014). The Maastricht Study: An extensive phenotyping study on determinants of type 2 diabetes, its complications and its comorbidities. European Journal of Epidemiology, 29(6), 439-451. PubMed ID: 24756374 doi:10.1007/s10654014-9889-0

Shiroma, E.J., Schepps, M.A., Harezlak, J., Chen, K.Y., Matthews, C.E., Koster, A., ... Harris, T.B. (2016). Daily physical activity patterns from hip- and wrist-worn accelerometers. Physiological Measurement, 37(10), 1852-1861. PubMed ID: 27654140 doi:10.1088/0967$3334 / 37 / 10 / 1852$
Sievänen, H., \& Kujala, U.M. (2017). Accelerometry-Simple, but challenging. Scandinavian Journal of Medicine \& Science in Sports, 27(6), 574-578. PubMed ID: 28466474 doi:10.1111/sms.12887

Suni, J.H., Oja, P., Laukkanen, R.T., Miilunpalo, S.I., Pasanen, M.E., Vuori, I.M., ... Bös, K. (1996). Health-related fitness test battery for adults: Aspects of reliability. Archives of Physical Medicine and Rehabilitation, 77(4), 399-405. PubMed ID: 8607767 doi:10.1016/ S0003-9993(96)90092-1

Tremblay, M.S., Aubert, S., Barnes, J.D., Saunders, T.J., Carson, V., Latimer-Cheung, A.E., ... SBRN Terminology Consensus Project Participants. (2017). Sedentary behavior research network (SBRN)_ Terminology consensus project process and outcome. International Journal of Behavioral Nutrition and Physical Activity, 14(1), 75. doi:10.1186/s12966-017-0525-8

Vähä-Ypyä, H., Husu, P., Suni, J., Vasankari, T., \& Sievänen, H. (2018). Reliable recognition of lying, sitting and standing with a waist-worn accelerometer. Scandinavian Journal of Medicine \& Science in Sports, 28(3), 1092-1102. PubMed ID: 29144567 doi:10.1111/ sms. 13017

Vähä-Ypyä, H., Vasankari, T., Husu, P., Mänttäri, A., Vuorimaa, T., Suni, J., \& Sievänen, H. (2015). Validation of cut-points for evaluating the intensity of physical activity with accelerometry-based mean amplitude deviation (MAD). PLoS One, 10(8), e0134813. doi:10.1371/ journal.pone.0134813

van der Velde, J.H.P.M., Koster, A., van der Berg, J.D., Sep, S.J.S, van der Kallen, C.J.H., Dagnelie, P.C., .. Savelberg, H.H.C.M. (2017). Sedentary behavior, physical activity, and fitness - the maastricht study. Medicine and Science in Sports and Exercise, 49(8), 15831591. PubMed ID: 28319587 doi:10.1249/MSS.0000000000001262

Vasankari, V., Husu, P., Vähä-Ypyä, H., Suni, J., Tokola, K., Halonen, J., ... Vasankari, T. (2017). Association of objectively measured sedentary behavior and physical activity with cardiovascular disease risk. European Journal of Preventive Cardiology, 24(12), 13111318. PubMed ID: 28530126 doi:10.1177/2047487317711048

Wennman, H., Pietilä, A., Rissanen, H., Valkeinen, H., Partonen, T., MäkiOpas, T., \& Borodulin, K. (2019). Gender, age and socioeconomic variation in 24-hour physical activity by wrist-worn accelerometers: The FinHealth 2017 Survey. Scientific Reports, 9(1), 6534. PubMed ID: 31024038 doi:10.1038/s41598-019-43007-x

Willetts, M., Hollowell, S., Aslett, L., Holmes, C., \& Doherty, A. (2018). Statistical machine learning of sleep and physical activity phenotypes from sensor data in 96220 UK Biobank participants. Scientific Reports, 8(1), 7961. PubMed ID: 29784928 doi:10.1038/s41598018-26174-1

Zou, D., Wennman, H., Ekblom, Ö., Grote, L., Arvidsson, D., Blomberg, A., ... Hedner, J. (2019). Insomnia and cardiorespiratory fitness in a middleaged population: The SCAPIS pilot study. Sleep \& Breathing, 23(1), 319-326. PubMed ID: 30547350 doi:10.1007/s11325-018-1765-9 